\title{
Atopic Dermatitis Pathogenesis: Lessons From Immunology
}

\author{
Luis F Santamaria-Babí1
}

1 Translational Immunology, Department of Cellular Biology, Physiology and Immunology, Faculty of Biology, University of Barcelona, Parc Científic de Barcelona, Barcelona, Spain

Key words: atopic dermatitis, translational research, homing, CLA

Abbreviations: CLA: Cutaneous Lymphocyte-Associated Antigen; Sa: Staphylococcus aureus; AD: atopic dermatitis

Citation: Santamaria-Babí L.F. Atopic dermatitis pathogenesis: lessons from immunology. Dermatol Pract Concept. 2022;12(1):e2022152. DOI: https://doi.org/10.5826/dpc.1201a152

Accepted: December 14, 2021; Published: January 2022

Copyright: (02022 Santamaria-Babi. This is an open-access article distributed under the terms of the Creative Commons Attribution-NonCommercial License (BY-NC-4.0), https://creativecommons.org/licenses/by-nc/4.0/, which permits unrestricted noncommercial use, distribution, and reproduction in any medium, provided the original authors and source are credited.

Funding: None.

Competing interests: None.

Corresponding author: Luis F Santamaria-Babí, PhD, Professor of Clinical and Translational Immunology. Translational Immunology. Parc Científic de Barcelona, Barcelona, Spain. E-mail: Luis.santamaria@ub.edu

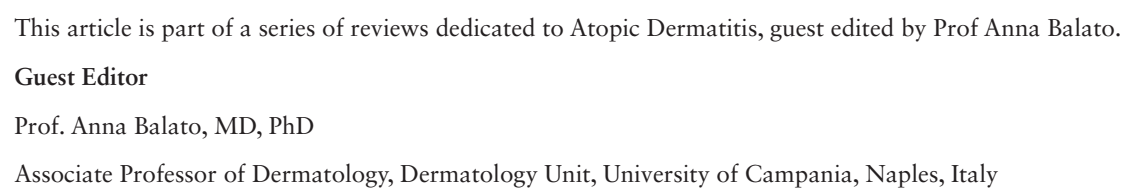

ABSTRACT Translational research has changed the understanding of atopic dermatitis (AD) pathogenesis beyond the basic mechanisms of immunology. The study in patients of rational therapies based on targeted therapies (biologicals) provides valuable information from the patient and provides lessons of clinical immunology on clinically relevant mechanism of $\mathrm{AD}$ pathogenesis. $\mathrm{AD}$ features such as skin barrier defect, skin dysbiosis, and pruritus share a common abnormal adaptive immune response process. Skin-homing CLA+CD4+ memory T-cells produce IL-4, IL-13, and IL-31 which are key mediators in $\mathrm{AD}$ pathogenesis. Lessons learned from $\mathrm{AD}$ show that translational immunology allows generating rational therapies for $\mathrm{AD}$ and learning its immunopathogenesis in the patient. 


\section{Introduction}

Atopic dermatitis $(\mathrm{AD})$ is a chronic inflammatory skin disease characterized by a defective epidermal barrier, cutaneous dysbiosis, pruritus, in a context of abnormal immune response [1]. The translational research model, originally applied to plaque psoriasis, has revolutionized the concepts of moderate to severe $\mathrm{AD}$ pathogenesis and has provided relevant clinical information on the important immunological mechanisms of $\mathrm{AD}$ [2]. Translational research allows validating in the patient new pathological mechanisms proposed in basic research. The use of targeted therapies such as humanized monoclonal antibodies directed to human targets (cytokines, receptors, cells...) in clinical trials, together with advanced technologies analyzing molecular mechanisms in patients (transcriptomics, proteomics. ..), has demonstrated that $\mathrm{AD}$ is a complex and heterogeneous condition that can now be treated more effectively with rational therapies directed to the pathogenic basis of the disease. The "omics" revolution facilitated the understanding of $\mathrm{AD}$ pathogenesis at molecular lever beyond clinical scores. Now all clinical studies with innovative drug therapies incorporate this molecular phenotyping of patients [3]. This review highlights, from a translational immunology perspective, recently identified immunological mechanisms involved in moderate to severe $\mathrm{AD}$ pathogenesis that are clinically relevant (Table 1 ).

\section{Type 2 (T2) Immune Response in AD}

Many AD patients exhibit an exaggerated immune T2 response that leads to increased $\mathrm{IgE}$ levels. The T2 immune response consists of the production of IL- 4 , IL-13, and IL- 5 by memory CD4+ Th2 cells, type-2 innate lymphoid cells (ILC2), mast cells, and basophils [4]. Thus, T2 immune response includes adaptive and innate mechanisms. The adaptive response is based on the capture of allergens, antigens, and superantigens by antigen presenting cells and presentation to specific CD4 memory T-cells that are activated and secrete IL-13, IL-4, IL-31, and IL-22 (Figure 1). On the other hand, the innate response is based on ILC2 cells that are tissue-resident and produce IL-5 and IL-13. To secrete cytokines, ILC2 lymphocytes need to be activated by epithelial cytokines, also termed alarmins, produced by keratinocytes. The best characterized alarmins are TLSP (thymic stromal lymphopoietin, IL-25, IL-33, and IL-1 $\alpha$ [1]. They have been very well studied also in $\mathrm{AD}[5,6]$.

\section{Th2 Response and Translational Research in AD}

IL-13 and IL4 have been demonstrated to be clinically relevant in $\mathrm{AD}$ by targeted therapeutic strategies since neutralization of IL-4R $\alpha$ and IL-13 clearly improved AD by inhibiting IL4/IL-13 and IL-13 biological activities, respectively [7]. In recent years, several clinical trials with biological therapies have sought to explore the relevance of Thymic stromal lymphoietin (TSLP), IL-25, IL-33 as possible new therapeutic targets for AD since they induce T2 response in ILC2. Lessons from these clinical trials that explore the roles of those alarmins in AD patients indicate that the neutralization of TSLP, IL-25 and IL-33 is not effective in improving disease [8]. In contrast to $\mathrm{AD}$, in asthma the clinical importance of IL-33 and TSLP is well demonstrated [9]. Since T2 cytokines can also be produced by CD4+ memory T-cells, at the present time, clinical efficacy in patients supports more strongly the relevance of the adaptive immune response in contrast to the innate response. In fact, CD4+ memory Th2 cells are the most abundant in inflamed lesions and considered the most important [1]. Interestingly, blockage of antigen presentation to

Table 1. Targeted Therapies in Moderate-to-Severe Atopic Dermatitis.

\begin{tabular}{|l|l|l|c|}
\multicolumn{1}{|c|}{ Target } & \multicolumn{1}{|c|}{ Mechanism } & \multicolumn{1}{|c}{ Therapy } & Clinical evidence in AD \\
\hline IL-4RA & Th2 (IL-4, IL-13) & Anti-IL-4R $\alpha$ & + \\
\hline IL-13 & IL-13 & Anti-IL-13 & + \\
\hline IL-31 & IL-31 & Anti-IL-31RA & + \\
\hline IL-22 & IL-22 & Anti-IL-22 & + \\
\hline OX40L & Antigen presentation & Anti-OX40L & + \\
\hline CCR4 & $\begin{array}{l}\text { CCR4 } \text { mediated CLA+ T-cell chemotaxis by } \\
\text { CCL17 and CCL22 }\end{array}$ & $\begin{array}{l}\text { CCR4 antagonist small } \\
\text { molecule }\end{array}$ & - \\
\hline IL-17A & IL-17A & Anti-IL-17A & - \\
\hline IL-5 & Eosinophil biology & Anti-IL-5 & - \\
\hline TSLP & Innate T2 response. TSLP activation of ILC2 & Anti-TSLP & - \\
\hline IL-33 & Innate T2 response. IL-33 activation of ILC2 & Anti-IL-33 & - \\
\hline IL-25 & Innate T2 response. IL-25 activation of ILC2 & Anti-IL-25 & - \\
\hline IL-23 & IL-23/Th17 axis & Anti-IL-23p19 & \\
\hline
\end{tabular}

TSLP = Thymic stromal lymphopoietin. 


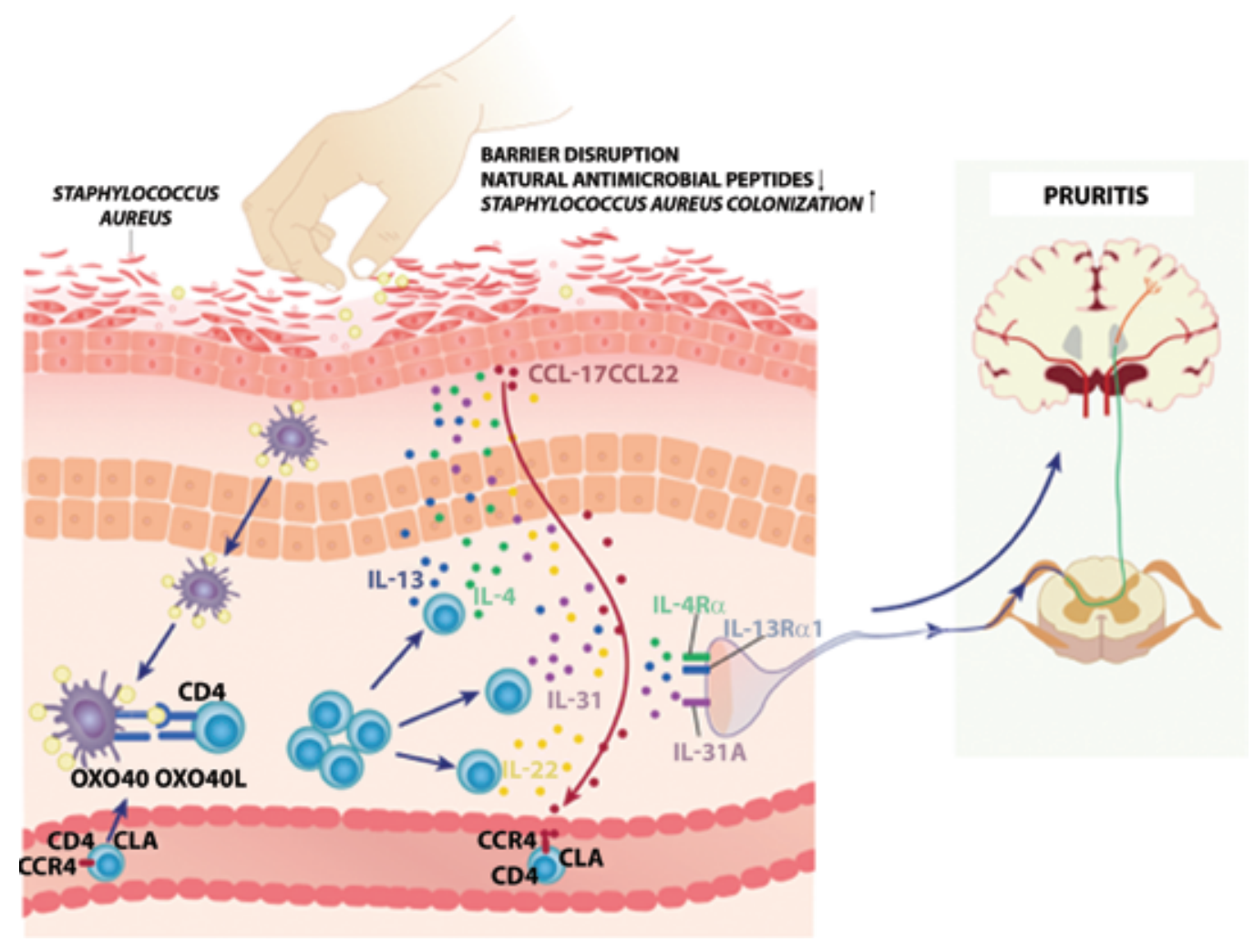

Figure 1. Lesson learned from translational immunology in atopic dermatitis (AD). The skin barrier abnormality, together with the itch-scratching cycle in $\mathrm{AD}$, facilitate skin entry of allergens and Staphylococcus aureus that active pathogenic skin-homing CLA+CD4+CCR4+ memory T-cells leading to production of IL-13, IL-4, IL-31, and IL-22. Th2 cytokines further alter the skin barrier, decreasing natural antimicrobial peptides that promote Staphylococcus aureus colonization. Cytokines IL-4, IL-13, and IL-31 bind to their respective receptors present in sensitive neurons in the skin that transmit pruritus to the central nervous system. Inflammation is amplified by IL-13-mediated infiltration of CLA+CD4+CCR4+ T cells attracted by IL-13-induced CCL17 production by keratinocytes.

CD4+ memory T-cells by a humanized monoclonal antibody that inhibits the OX-OX40L interaction has demonstrated to be effective in the clinic (Figure 1) [10]). IL-5 constitutes another T2 cytokine that regulates eosinophil generation and survival. In AD, neutralization of IL-5 has not provided clinical improvement in patients, whereas in asthma, it constitutes a biological therapy already approved [8].

\section{Novel Insights on IL-13 Relevance in AD}

IL-4 and IL-13 represent the best clinically validated cytokines in AD due to the efficacy of biologicals targeting IL-4RA and IL-13. Recent studies in AD lesional skin indicate that IL-13 may have more relevance in the inflammatory processes that take place in the skin than IL-4 [7]. IL-13 receptors, IL-13RA1 and IL-13RA2, are preferentially found in non-hematopoietic cells such as keratinocytes and fibroblasts. In addition, IL-13 it is expressed in higher level in AD lesions compared to IL-4, and transcriptomic analyses have demonstrated a dominant IL-13 molecular signature [11]. Interestingly, JAK1 is the most highly expressed member of the JAK family in lesional AD biopsies and is involved in IL-13 signal transduction [12]. Besides this, IL-13 also plays a relevant role in the recruitment of pathogenic skin-homing memory T-cells that express the cutaneous lymphocyte-associated antigen (CLA) positive on their surface $[1,13]$. IL-13 and CCR4 are closely related in AD inflammation. The CCR4 is a chemokine receptor preferentially expressed by CLA+CD4+ T-cells [14] whose ligands are CCL17 and CCL22 and are induced in keratinocytes upon IL-13 activation and preferentially attract CLA+ CD4+ lymphocytes [15]. Interestingly, CCL17 is considered a relevant biomarker for $\mathrm{AD}$ [16] and a recent study has shown that a small molecule antagonist of the CCR4 receptor can improve AD [17].

\section{IL-13 and IL-4 Affect the Skin Barrier Function and the Microbial Cutaneous Colonization by Staphyloccocus Aureus in AD}

The skin barrier integrity is damaged in $\mathrm{AD}$ cutaneous lesions making the stratum corneum permeable to allergens, microbes, and their toxins. Besides genetic structural defects, such as filaggrin mutations, which are present in some $\mathrm{AD}$ patients, IL-13 and IL-4 reduce the expression of relevant skin elements involved in the skin barrier such as filaggrin, loricrin, involucrin and important lipid components [18,19]. By far, Staphylococcus aureus (Sa) is the best characterized microorganism in $\mathrm{AD}$ because it is present on the skin of $\mathrm{AD}$ patients [20]. In fact, Sa skin-colonized AD patients have been recently shown to present a more severe form of disease than 
non-colonized ones [21]. Th2 cytokines have been shown to facilitate skin colonization by Sa since they reduce the expression of natural antimicrobial peptides, thus reducing defenses against Sa infection, favoring Sa skin adherence by promoting fibronectin production [20].

\section{Immunological Mechanisms of Pruritus}

Itch is probably the symptom that most affects the quality of life of $\mathrm{AD}$ patients, and it is part of the itch-scratching cycle that most patients suffer from [22]. The adaptive immune response is responsible to a great extent of the pruritus present in $\mathrm{AD}$ as demonstrated by biological therapies and small molecules that inhibit key signaling pathways involved in pruritus. There are 2 different immunological mechanisms behind the adaptive immune response and signal transduction of itch by sensitive nervous fibers in the skin. CD $4+$ Th 2 cells producing IL-4 and IL-13, and the Th2 related IL-31. These 3 cytokines stimulate sensitive cutaneous fibers that express their specific receptors to transmit pruritus to the central nervous system through JAK1 kinase signaling [23]. The relevance of IL-4 and IL-13 in pruritus is supported by the important release of itch by neutralizing antibodies to IL-4RA and IL-13 in moderate to severe patients. In addition, small molecules that act as JAK inhibitors and block JAK1 signaling of IL-4 and IL-13 receptors, are also potent therapies that act on immune-mediated itch. IL-31 is a unique cytokine since it is mainly involved in pruritus generation. CLA+CD4+ memory T-cells constitute the main source of IL-31 [24]. IL-31 binds to the IL-31RA that it is expressed in cutaneous sensitive neurons and transduces signaling through JAk1 signaling. The relevance of IL-31 in itch in AD patients is supported by the antipruritic activity of anti-IL-31RA which is currently in phase III trials [25].

\section{Immunological Response In AD: Beyond Th2 and Th1}

The classical model of AD pathogenesis where the acute phase is Th2 and the chronic stage is Th1 has evolved to more a complex scenario including Th1, Th2, Th17, and Th22 cytokines [26]. However, the presence of all those T-cell subtypes in AD does not imply they are clinically relevant players. At present, translational research has demonstrated that only some of them are important, although it cannot be discarded that in different AD phenotypes, endotypes, or ethnicities these cytokines may be relevant. IL-22, IL-17A and IL-23 are well characterized mediators of inflammation in psoriasis; however, they are also present in AD inflammation. The study of specific antibodies for these cytokines in AD patients has clarified their role in this disease. Neutralization of IL-22 improved AD preferentially in patients with increased lesional expression of IL-22 at baseline [27]. Current efforts to demonstrate the clinical relevance of IL-17A and IL-23 have failed since neutralization of those cytokines in patients has not provided any clinical benefit [8]. In relation to the immune response, new technologies in simultaneous quantification of serum biomarkers of immune response have allowed to classify moderate to severe $\mathrm{AD}$ patients into 4 endotypes which demonstrate patients heterogeneity. These are IL-1R1 and skin-homing tropism, dominant Th1/Th2/Th17 response, Th2/Th22/CCl18 response, and low levels of Th2 cytokines and eosinophilia [28].

\section{Circulating CLA+ Memory T Cells and AD}

Circulating T-cells expressing the CLA antigen represent a subset of memory T-cells that reflect cutaneous immunological abnormalities taking place in the skin [29]. They are used to understand atopic dermatitis phenotypes as possible cell biomarkers of disease [30,31]. The translational relevance of this subset in $\mathrm{AD}$, which also exists in human circulation as resident memory Th2 cells, as well as in the skin [32], has been further studied during dupilumab treatment of moderate to severe AD patients. At week 4 of treatment, dupilumab decreased IL-4, IL-13 and IL-22 production in $\mathrm{CLA}+\mathrm{CD} 4+\mathrm{CCR} 4+$, but not in the CLA- memory $\mathrm{T}$ cells subset $[33,34]$. These results indicate that circulating skin homing lymphocytes preferentially respond to IL4RA blockage in $\mathrm{AD}$ patients and are in line with the relevance of adaptive immune response in $\mathrm{AD}$ in the current model of $\mathrm{AD}[1]$.

\section{Conclusions}

Current translational immunology results support a simplified model of AD pathogenesis (Figure 1), where patients carry an abnormal immune response that reacts to agents accessing the skin trough a permeable skin barrier, in the context of the itch-scratching circle. Circulating CLA+CD4+CCR4+ memory T-cells infiltrate non-lesional skin and become activated by antigen presenting cells and secrete IL-4, IL-13, and IL-31 among other cytokines. IL-4 and IL-13, and in particular IL-13 due to the presence of the IL-13 receptor in the skin and greater amount of IL-13 than IL-4, alter skin barrier function. In addition, IL-13 would promote Sa colonization by decreasing natural antimicrobial peptides and adherence through increased production of fibronectin. Those 3 cytokines mediate pruritus by binding to their specific receptors in sensitive neurons that transmit pruritus to central nervous system. IL-13 would promote inflammation by the recruitment of skin-homing CLA+CD4+CCR4+ that are attracted by IL-13-activated keratinocytes that produce CCL17. 


\section{References}

1. Langan SM, Irvine AD, Weidinger S. Atopic dermatitis. Lancet. 2020;396(10247):345-360. DOI: 10.1016/S01406736(20)31286-1. PMID: 32738956.

2. Noda S, Krueger JG, Guttman-Yassky E. The translational revolution and use of biologics in patients with inflammatory skin diseases. J Allergy Clin Immunol. 2015;135(2):324-336. DOI: 10.1016/j.jaci.2014.11.015. PMID: 25541257.

3. Oliva M, Renert-Yuval Y, Guttman-Yassky E. The "omics" revolution: Redefining the understanding and treatment of allergic skin diseases. Curr Opin Allergy Clin Immunol. 2016;16(5):469476. DOI: 10.1097/ACI.0000000000000306. PMID: 27490125.

4. Lloyd CM, Snelgrove RJ. Type 2 immunity: Expanding our view. Sci Immunol. 2018 ;3(25):eaat1604. DOI: 10.1126/sciimmunol. aat1604. PMID: 29980619.

5. Saunders SP, Moran T, Floudas A, et al. Spontaneous atopic dermatitis is mediated by innate immunity, with the secondary lung inflammation of the atopic march requiring adaptive immunity. J Allergy Clin Immunol. 2016;137(2):482-491. DOI: 10.1016/j. jaci.2015.06.045. PMID: 26299987. PMCID: PMC4735016.

6. Salimi M, Barlow JL, Saunders SP, et al. A role for IL-25 and IL33-driven type-2 innate lymphoid cells in atopic dermatitis. J Exp Med. 2013;210(13):2939-2950. DOI: 10.1084/jem.20130351. PMID: 24323357. PMCID: PMC3865470.

7. Bieber T. Interleukin-13: Targeting an underestimated cytokine in atopic dermatitis. Allergy. 2020;75(1):54-62. DOI: 10.1111/ all.13954. PMID: 31230370.

8. Bieber T. Atopic dermatitis: an expanding therapeutic pipeline for a complex disease. Nat Rev Drug Discov. 2022;21(1):21-40. DOI: 10.1038/s41573-021-00266-6. PMID: 34417579. PMCID: PMC8377708.

9. Tamari M, Trier AM, Kim BS. Emerging targeted therapeutics underscore immunologic heterogeneity of asthma. J Allergy Clin Immunol. 2021;148(3):719-721. DOI: 10.1016/j.jaci.2021.07.008. PMID: 34310926.

10. Guttman-Yassky E, Pavel AB, Zhou L, et al. GBR 830, an anti-OX40, improves skin gene signatures and clinical scores in patients with atopic dermatitis. J Allergy Clin Immunol. 2019;144(2):482-493.e7. DOI: 10.1016/j.jaci.2018.11.053. PMID: 30738171.

11. Tsoi LC, Rodriguez E, Degenhardt F, et al. Atopic Dermatitis Is an IL-13-Dominant Disease with Greater Molecular Heterogeneity Compared to Psoriasis. J Invest Dermatol. 2019;139(7):14801489. DOI: 10.1016/j.jid.2018.12.018. PMID: 30641038: PMCID: PMC6711380.

12. Möbus L, Rodriguez E, Harder I, et al. Atopic dermatitis displays stable and dynamic skin transcriptome signatures. $J$ Allergy Clin Immunol. 2021;147(1):213-223. DOI: $10.1016 / \mathrm{j}$. jaci.2020.06.012. PMID: 32615169.

13. Weidinger S, Beck LA, Bieber T, Kabashima K, Irvine AD. Atopic dermatitis. Nat Rev Dis Primers. 2018;4(1):1. DOI: 10.1038/ s41572-018-0001-z. PMID: 29930242.

14. Campbell JJ, Haraldsen G, Pan J, et al. The chemokine receptor CCR4 in vascular recognition by cutaneous but not intestinal memory T cells. Nature. 1999;400(6746):776-780. DOI: 10.1038/23495. PMID: 10466728.

15. Vestergaard C, Bang K, Gesser B, Yoneyama H, Matsushima K, Larsen CG. A Th2 chemokine, TARC, produced by keratinocytes may recruit CLA+CCR4+ lymphocytes into lesional atopic dermatitis skin. J Invest Dermatol. 2000;115(4):640-646. DOI: 10.1046/j.1523-1747.2000.00115.x. PMID: 10998136.

16. Renert-Yuval Y, Thyssen JP, Bissonnette R, et al. Biomarkers in atopic dermatitis-a review on behalf of the International Eczema Council. J Allergy Clin Immunol. 2021;147(4):1174-1190.e1. DOI: 10.1016/j.jaci.2021.01.013. PMID: 33516871.

17. Bissonnette R, Rulloda J, Nadine Lee N, et al. RPT193, an oral CCR4 inhibitor: Efficacy results from a randomized, placebo-controlled Phase $1 \mathrm{~b}$ monotherapy trial in patients with moderate-tosevere atopic dermatitis. ISDS 2021. Abstract 88.

18. Kim BE, Leung DYM, Boguniewicz M, Howell MD. Loricrin and involucrin expression is down-regulated by Th2 cytokines through STAT-6. Clin Immunol. 2008;126(3):332-337. DOI: 10.1016/j. clim.2007.11.006. PMID: 18166499: PMCID: PMC2275206.

19. Howell MD, Kim BE, Gao P, et al. Cytokine modulation of atopic dermatitis filaggrin skin expression. J Allergy Clin Immunol. 2009;124(3 Suppl 2):R7-R12. DOI: 10.1016/j.jaci.2009.07.012. PMID: 19720210.

20. Kim J, Kim BE, Ahn K, Leung DYM. Interactions between atopic dermatitis and staphylococcus aureus infection: Clinical implications. Allergy Asthma Immunol Res. 2019;11(5):593-603. DOI: 10.4168/aair.2019.11.5.593. PMID: 31332972. PMCID: PMC6658404.

21. Simpson EL, Villarreal M, Jepson B, et al. Patients with Atopic Dermatitis Colonized with Staphylococcus aureus Have a Distinct Phenotype and Endotype. J Invest Dermatol. 2018;138(10):22242233. DOI: 10.1016/j.jid.2018.03.1517. PMID: 29604251. PMCID: PMC6153055.

22. Mack MR, Kim BS. The Itch-Scratch Cycle: A Neuroimmune Perspective. Trends Immunol. 2018;39(12):980-991. DOI: 10.1016/j. it.2018.10.001. PMID: 30471983.

23. Oetjen LK, Mack MR, Feng J, et al. Sensory Neurons Co-opt Classical Immune Signaling Pathways to Mediate Chronic Itch. Cell. 2017;171(1):217-228.e13. DOI: 10.1016/j.cell.2017.08.006. PMID: 28890086. PMCID: PMC5658016.

24. Bilsborough J, Leung DYM, Maurer M, et al. IL-31 is associated with cutaneous lymphocyte antigen-positive skin homing $\mathrm{T}$ cells in patients with atopic dermatitis. J Allergy Clin Immunol. 2006;117(2):418-425. DOI: 10.1016/j.jaci.2005.10.046. PMID: 16461143.

25. Silverberg JI, Pinter A, Alavi A, et al. Nemolizumab is associated with a rapid improvement in atopic dermatitis signs and symptoms: subpopulation (EASI $\geq 16$ ) analysis of randomized phase 2B study. J Eur Acad Dermatol Venereol. 2021;35(7):1562-1568. DOI: 10.1111/jdv.17218. PMID: 33711179.

26. Gittler JK, Shemer A, Suárez-Fariñas M, et al. Progressive activation of TH2/TH22 cytokines and selective epidermal proteins characterizes acute and chronic atopic dermatitis. $J \mathrm{Al}$ lergy Clin Immunol. 2012;130(6):1344-1354. DOI: 10.1016/j. jaci.2012.07.012. PMID: 22951056. PMCID: PMC3991245.

27. Brunner PM, Pavel AB, Khattri S, et al. Baseline IL-22 expression in patients with atopic dermatitis stratifies tissue responses to fezakinumab. J Allergy Clin Immunol. 2019;143(1):142-154. DOI: 10.1016/j.jaci.2018.07.028. PMID: 30121291.

28. Bakker DS, Nierkens S, Knol EF, et al. Confirmation of multiple endotypes in atopic dermatitis based on serum biomarkers. $J$ Allergy Clin Immunol. 2021;147(1):189-198. DOI: 10.1016/j. jaci.2020.04.062. PMID: 32526312.

29. de Jesús-Gil C, Sans-de SanNicolàs L, García-Jiménez I, et al. The Translational Relevance of Human Circulating Memory 
Cutaneous Lymphocyte-Associated Antigen Positive T Cells in Inflammatory Skin Disorders. Front Immunol. 2021;12:652613. DOI: 10.3389/fimmu.2021.652613. PMID: 33833765. PMCID: PMC8021783.

30. Ferran M, Santamaria-Babi LF. Pathological mechanisms of skin homing T cells in atopic dermatitis. World Allergy Organ J. 2010;3(3):44-47. DOI: 10.1097/WOX.0b013e3181d675f8. PMID: 23282416. PMCID: PMC3651146.

31. Czarnowicki T, Santamaria-Babí LF, Guttman-Yassky E. Circulating CLA+ T cells in atopic dermatitis and their possible role as peripheral biomarkers. Allergy. 2017;72(3):366-372. DOI: 10.1111/all.13080. PMID: 27861978.

32. Strobl J, Gail L, Kleissl L, et al. Human resident memory T cells exit the skin and mediate systemic Th2-driven inflamma- tion. J Exp Med. 2021;218(11):e20210417. DOI: 10.1084/ jem.20210417. PMID: 34643646. PMCID: PMC8563284.

33. Bakker DS, van der Wal MM, Heeb LEM, et al. Early and LongTerm Effects of Dupilumab Treatment on Circulating T-Cell Functions in Patients with Moderate-to-Severe Atopic Dermatitis. J Invest Dermatol. 2021;141(8):1943-1953.e13. DOI: 10.1016/j. jid.2021.01.022. PMID: 33610558.

34. Roesner LM, Bridgewood C, McGonagle D, Wittmann M. Dupilumab: An Opportunity to Unravel In Vivo Actions of IL-4 and IL-13 in Humans. J Invest Dermatol. 2021;141(8):1879-1881. DOI: 10.1016/j.jid.2021.02.007. PMID: 34303467. 